\title{
Study and application of the complex, spectral and polarization modulation in liquid crystal modulators
}

\section{Estudio y aplicación de la modulación compleja, espectral y de polarización en moduladores de cristal líquido}

\author{
José Luis Martínez Fuentes $\left(^{*}, S\right)$ \\ Departamento de Ciencia de Materiales Óptica y Tecnología Electrónica, Universidad Miguel Hernández de \\ Elche, Avda. Universidad s/n, 03202, Elche, Alicante, Spain. \\ (*) Email: jose.martinez@umh.es \\ S: miembro de SEDOPTICA / SEDOPTICA member \\ Received / Recibido: 24/10/2014. Revised / Revisado: 11/11/2014. Accepted / Aceptado: 12/11/2014. \\ DOI: http://dx.doi.org/10.7149/OPA.47.4.281
}

\begin{abstract}
:
This work summarizes the doctoral thesis with the same title. Concretely, the following topics are treated: 1) the application of a microscopic Jones matrix model to predict the spectral and colorimetric modulation in TN-LCD spatial light modulators, 2) the extended phase depth modulation phenomenon in such devices, 3) the study of the complex modulation of modulators by means of Panchartnam's phase, and 4) some experimental set-ups developed during the thesis.
\end{abstract}

Key words: Liquid Crystal Modulators, Color Modulation, Complex Modulation, Pancharatnam's Phase.

\section{RESUMEN:}

Este trabajo presenta el resumen de la tesis doctoral con el mismo título. En concreto, se tratan los siguientes aspectos: 1) la aplicación de un modelo microscópico de matrices de Jones para la predicción de la modulación espectral y colorimétrica de pantallas TN-LCD, 2) el fenómeno de la modulación extendida de fase en pantallas TN-LCD, 3) el estudio de la modulación compleja de moduladores mediante la fase de Pancharatnam y 4) algunos sistemas experimentales desarrollados en la tesis.

Palabras clave: Moduladores de Cristal Líquido, Modulación del Color, Modulación Compleja, Fase de Pancharatnam.

\section{REFERENCES AND LINKS / REFERENCIAS Y ENLACES}

[1]. J. L. Martínez Fuentes, Estudio y Aplicación de la Modulación Compleja, Espectral y de Polarización en Moduladores de Cristal Líquido, PhD Thesis, Universidad Miguel Hernández de Elche, Spain (2012).

[2]. A. Márquez, J. Campos, M. J. Yzuel, I. Moreno, J. A. Davis, C. Iemmi, A. Moreno, A. Robert, "Characterization of edge effects in twisted nematic liquid crystal displays", Opt. Eng. 39, 3301-3307 (2000). DOI

[3]. J. L. Martínez, P. García-Martínez, M. M. Sánchez-López, I. Moreno, “Accurate color predictability based on a spectral retardance model of a twisted-nematic liquid-crystal display", Opt. Commun. 284, 2441-2447 (2011). DOI

[4]. J. L. Martínez, I. Moreno, J. A. Davis, T. J. Hernandez, K. P. McAuley, "Extended phase modulation depth in twisted nematic liquid crystal displays", Appl. Opt. 49, 5929-5937 (2010). DOI

[5]. J. M. Cabrera, F. Agulló López, F. J. López, Óptica Electromagnética, vol I: Fundamentos, Addison Wesley/Ediciones UA, Madrid, Spain (2000).

[6]. S. Pancharatnam, "Generalized theory of interference, and its applications. Part I. Coherent pencils", P. Ind. Acad. Sci. A 44, 247-262 (1956). 
[7]. M. V. Berry, "The adiabatic phase and Pancharatnam's phase for polarized light", J. Mod. Optic 34, 1401-1407 (1987). DOI

[8]. J. L. Martínez Fuentes, J. Albero, I. Moreno, "Analysis of optical polarization modulation systems through the Pancharatnam connection", Opt. Commun. 285, 393-401 (2012). DOI

[9]. J. L. Martínez, A. Martínez-García, I. Moreno, "Wavelength-compensated color Fourier diffractive optical elements using a ferroelectric liquid crystal on silicon display and a color-filter wheel", Appl. Opt. 48, 911-918 (2009). DOI

[10]. J. Otón, P. García-Martínez, I. Moreno, J. García, "Phase joint transform correlator dor nonlinear binary correlations", Opt. Commun. 245, 113-124 (2004). DOI

[11].P. García-Martínez, J. L. Martínez, M. M. Sánchez-López, I. Moreno, "Wavelength-compensated timesequential multiplexed color joint transform correlator", Appl. Opt. 49, 4866-4873 (2010). DOI

[12].D. Engström, A. Frank, J. Backsten, M. Goksör, J. Bengtsson, "Grid-free multiple spot generation with an efficient single-plane FFT-based algorithm", Opt. Express 17, 9989-10000 (2009). DOI

[13]. H. Dammann, E. Klotz, "Coherent generation and inspection of two-dimensional periodic structures", Opt. Acta 24, 505-515 (1977). DOI

[14]. I. Moreno, J. A. Davis, D. M. Cottrell, N. Zhang, X. C. Yuan, "Enconding generalized phase functions on Dammann gratings", Opt. Lett. 35, 1536-1538 (2010). DOI

[15]. J. A. Davis, I. Moreno, J. L. Martínez, T. J. Hernandez, D. M. Cottrell, "Creating three-dimensional lattice patterns using programmable Dammann gratings", Appl. Opt. 50, 3653-3657 (2011). DOI

[16].J. L. Martínez, I. Moreno, F. Mateos, "Hiding binary optical data with orthogonal circular polarizations", Opt. Eng. 47, 030504 (2008). DOI

[17].M. L. Álvarez, A. Márquez, L. A. Puerta, R. Estévez, E. Fernández, A. Beléndez, I. Pascual, "Caracterización de un display de cristal líquido de baja resolución para su uso como modulador óptico", Opt. Pura Apl. 40, 95-103 (2007).

[18]. R. C. Jones, "A new calculus for the treatment of optical systems", J. Opt. Soc. Am. A 31, 488-503 (1941). DOI

[19]. J. A. Davis, I. Moreno, P. Tsai, "Polarization eigenstates for twisted-nematic liquid-crystal displays", Appl. Opt. 37, 937-945 (1998). DOI

[20]. J. Nicolás, J. Campos, and M. J. Yzuel, "Phase and amplitude modulation of elliptic polarization states by nonabsorbing anisotropic elements: application to liquid-crystal devices", J. Opt. Soc. Am. A 19, 1013-1020 (2002). DOI

[21].J. A. Davis, J. Nicolás, A. Márquez, "Phasor analysis of eigenvectors generated in liquid-crystal displays", Appl. Opt. 41, 4579-4584 (2002). DOI

[22].Y. Aharonov, J. Anandan, "Phase change during a cyclic quantum evolution", Phys. Rev. Lett. 58, 15931596 (1987). DOI

[23]. H. Schmitzer, S. Klein, W. Dultz, "An experimental test of the path dependency of Pancharatnam's geometric phase", J. Mod. Opt. 45, 1039-1047 (1998). DOI

[24]. J. Courtial, "Wave plates and the Pancharatnam phase", Opt. Commun. 171, 179-183 (1999). DOI

[25]. M. Skeren, I. Ritcher, P. Fiala, "Iterative Fourier transform algorithm: Comparison of various approaches", J. Mod. Opt. 49, 1851-1870 (2002). DOI

[26]. R. L. Eriksen, P. C. Mogensen, J. Glückstad, "Elliptical polarisation encoding in two dimensions usign phase-only spatial light modulators", Opt. Commun. 187, 325-336 (2001). DOI

\section{Introducción}

La Tesis Doctoral que aquí se resume [1] se ha centrado en el estudio de los aspectos de la modulación óptica en moduladores espaciales de luz de cristal líquido y su aplicación en la construcción de diversos sistemas experimentales con estos dispositivos. Este trabajo ha permitido obtener la tesis doctoral denominada: "Estudio y aplicación de la modulación compleja, espectral y de polarización en moduladores de cristal líquido", realizada en la modalidad de conjunto de publicaciones, y que fue finalmente defendida el día 1 de Junio de 
2012 en la Universidad Miguel Hernández de Elche.

Los aspectos teóricos del trabajo son los que se detallan a continuación. En primer lugar, la inclusión de modelos de dispersión cromática en el modelo de Márquez [2] para moduladores TNLCD (del inglés Twisted Nematic Liquid Crystal Display) con objeto de realizar predicciones espectrales y colorimétricas [3]. La aplicación de este modelo se presenta en el apartado 2 de este resumen. Otro de los aspectos teóricos abarca el estudio del fenómeno de la modulación extendida de fase [4] en los sistemas de modulación compleja con pantallas de cristal líquido nemático rotado o TN-LCD. Este fenómeno se detalla en el apartado tercero. El tercer aspecto a destacar comprende el desarrollo de una representación gráfica en la esfera de Poincaré [5] y basada en la fase geométrica o fase de Pancharatnam [6,7] que nos ha permitido estudiar los sistemas de modulación compleja con moduladores de cristal líquido [8]. Esta teoría se resume en el apartado cuarto de este trabajo.

Por otro lado, los sistemas experimentales desarrollados en este trabajo comprenden: un sistema de transformada de Fourier en color basado en un esquema de multiplexado temporal de la longitud de onda y una rueda de filtros de color [9], un sistema de correlación óptica [10] para detección de imágenes en color [11], un sistema para la generación de patrones tridimensionales de puntos [12-14] basado en el empleo de elementos difractivos de Dammann [15], un sistema de codificación de imágenes mediante estados de polarización [16] y finalmente un conjunto de sistemas electrónicos e informáticos para dar soporte a los sistemas experimentales y las simulaciones. Los principales resultados de todos estos sistemas experimentales se resumen brevemente en el quinto apartado de este escrito. Por último se presentan las conclusiones y agradecimientos.

\section{Aplicación de un modelo microscópico para la predicción espectral y colorimétrica de pantallas TN-LCD}

El modelo de Márquez de moduladores TNLCD [2] ha proporcionado resultados muy satisfactorios en la predicción de la modulación compleja [17], pero hasta ahora sólo se había aplicado para realizar predicciones con iluminación monocromática. Sin embargo, este modelo microscópico hace posible extender las propiedades de modulación a longitudes de onda diferentes de las de calibración mediante un ajuste de la dependencia espectral de la birrefringencia del material para realizar predicciones espectrales de la modulación compleja. Con esta información, es posible obtener las características colorimétricas $\mathrm{y}$, por tanto, predecir la modulación del color en base representaciones estándar como la CIE1931-xy.

\section{2.a. Modelo físico}

Las celdas de un TN-LCD están conformadas por moléculas de cristal líquido que van rotando desde la cara de entrada hasta la de salida de la celda y la luz a lo largo de ésta tiende a seguir la rotación del director (eje) molecular. Esta disposición se mantiene en ausencia de voltaje aplicado. Al aumentar el campo aplicado, las moléculas se alinean con éste en la mayor parte de la celda, salvo en las paredes laterales cercanas a los electrodos, en las cuales
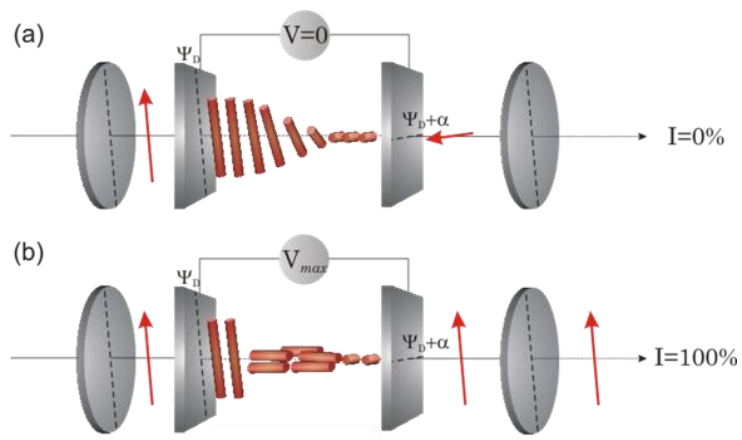

Fig. 1. Representación del las moléculas de cristal líquido en el interior de una celda de cristal líquido nemático rotado (TN-LCD) en función del voltaje aplicado a la celda: (a) en ausencia de voltaje aplicado y (b) cuando el voltaje es máximo. 
permanecen ancladas en mayor o menor grado. La Fig. 1(a) se representa las moléculas cuando el voltaje es nulo. La Fig. 1(b) muestra las moléculas cuando el voltaje es suficiente para inclinar completamente el director molecular.

Al modelar cada molécula como un desfasador microscópico y considerar el giro de éstas, es posible inferir una matriz de Jones [18] equivalente del TN-LCD, dada por:

$$
\begin{aligned}
\left.\mathbf{M}=e^{-i[\beta+2 \delta}\right] \mathbf{R}\left(-\alpha-\Psi_{D}\right) . \\
\\
\cdot\left[\begin{array}{cc}
X-i Y & Z \\
-Z & X+i Y
\end{array}\right] \mathbf{R}\left(\Psi_{D}\right)
\end{aligned}
$$

donde $\quad X=\cos \gamma \cos 2 \delta-(\beta / \gamma) \operatorname{sen} \gamma \operatorname{sen} 2 \delta$, $Y=\cos \gamma \operatorname{sen} 2 \delta+(\beta / \gamma) \operatorname{sen} \gamma \cos 2 \delta, \quad \mathrm{Z}=(\alpha / \gamma) \operatorname{sen} \gamma$, $\gamma^{2}=\alpha^{2}+\beta^{2}$. En estas expresiones, $\alpha$ es el ángulo de giro (conocido como twist) en el plano transversal de las moléculas, $\Psi_{D}$ es el ángulo del director en la cara de entrada, $\beta$ el desfase total de las moléculas en la zona interior de la celda y $\delta$ el desfase total de éstas en las caras de entrada y salida.

\section{2.b. Dependencia espectral}

Conociendo la posición del director, el twist y la evolución de los parámetros $\beta$ y $\delta$ con el voltaje, o equivalentemente el nivel de gris, y la longitud de onda se tendrá totalmente caracterizado el dispositivo. Para el valor máximo de beta (cuando el dispositivo está apagado), se supone una dispersión de Cauchy como la siguiente:

$$
\beta_{\text {off }}(\lambda)=\frac{a}{\lambda}+\frac{b}{\lambda^{3}},
$$

y para interpolar el valor de este parámetro y el de delta en encendido, se suponen válidas las siguientes relaciones:

$$
\begin{aligned}
& \beta(\lambda, g)=\beta\left(\lambda_{r}, g\right) \cdot \frac{\beta_{o f f}(\lambda)}{\beta_{o f f}\left(\lambda_{r}\right)}, \\
& \delta(\lambda, g)=\delta\left(\lambda_{r}, g\right) \cdot \frac{\beta_{o f f}(\lambda)}{\beta_{o f f}\left(\lambda_{r}\right)},
\end{aligned}
$$

donde $\lambda_{\mathrm{r}}$ es una de las longitudes de onda en las que se ha calibrado el dispositivo.

Las curvas de calibración obtenidas para un modulador XGA3 de CRL-OPTO, se muestran en la Fig. 2. La Fig. 2(a) muestra la dispersión de beta en apagado. Las Figs. 2(b) y 2(c) muestran la evolución de beta y delta con el nivel de gris para las longitudes de onda $633 \mathrm{~nm}, 514 \mathrm{~nm}$, $488 \mathrm{~nm}$ y $457 \mathrm{~nm}$, respectivamente.

\section{2.c. Resultados}

Usando esta información es posible predecir y optimizar la respuesta del dispositivo. Por ejemplo, la figura 3 se muestra una optimización para barrer la máxima área en el diagrama CIExy 1931 cuando el modulador se ilumina mediante una fuente de luz extensa, cuyo espectro se muestra en la Fig. 3(a), y se encierra entre dos polarizadores lineales. Las Figs. 3(b) y 3(c) muestran las curvas espectrales de intensidad y las coordenadas de color para distintos niveles de gris enviados al dispositivo. Puede apreciarse la buena concordancia entre las predicciones (líneas) y los valores experimentales (puntos). Esta configuración se obtiene al colocar el polarizador y el analizador a $19,5^{\circ}$ y $-29,5^{\circ}$ relativos al director del modulador, respectivamente.

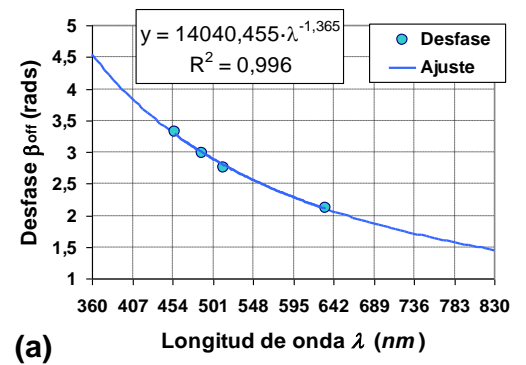

Fig. 2. (a) Dispersión del desfase $\beta_{\text {off }}$ en apagado. (b) $\beta$ y (b) $\delta$ en función del nivel de gris para $633 \mathrm{~nm}, 514 \mathrm{~nm}, 488 \mathrm{~nm}$ y $457 \mathrm{~nm}$, respectivamente.
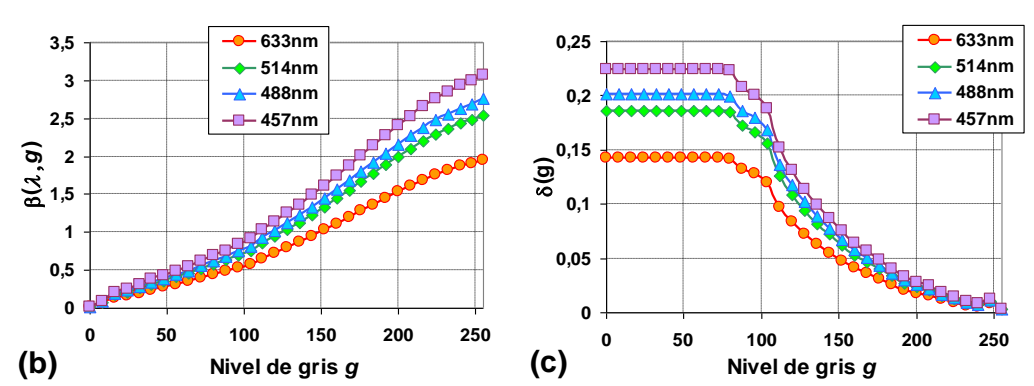

(c) 


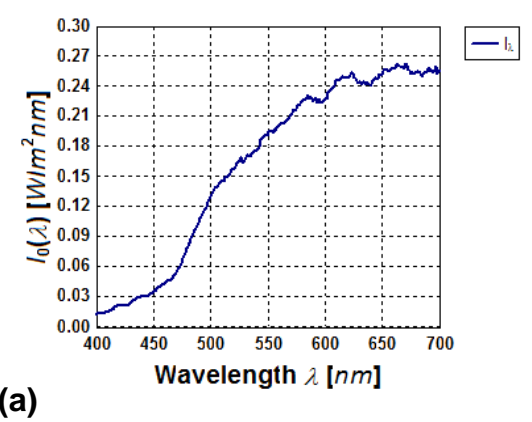

(a)

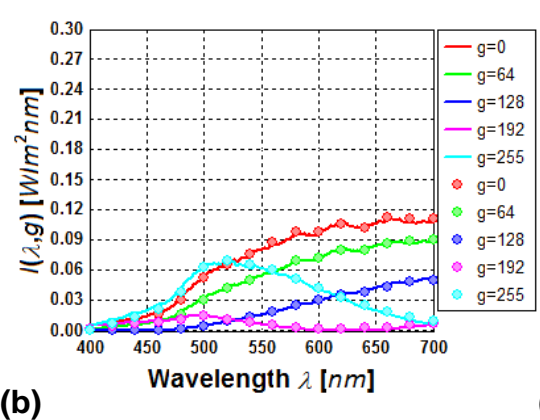

(b)

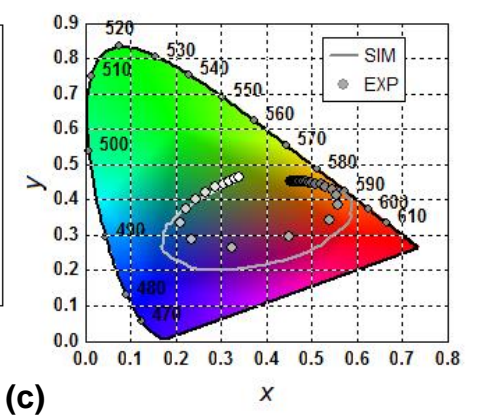

(c)

Fig. 3. (a) Espectro del iluminante, (b) intensidad en función de la longitud de onda y (c) coordenadas de color en el diagrama CIE-xy 1931 en función del nivel de gris para una configuración de polarizadores dada. Las líneas indican predicciones y lo puntos valores experimentales [3].

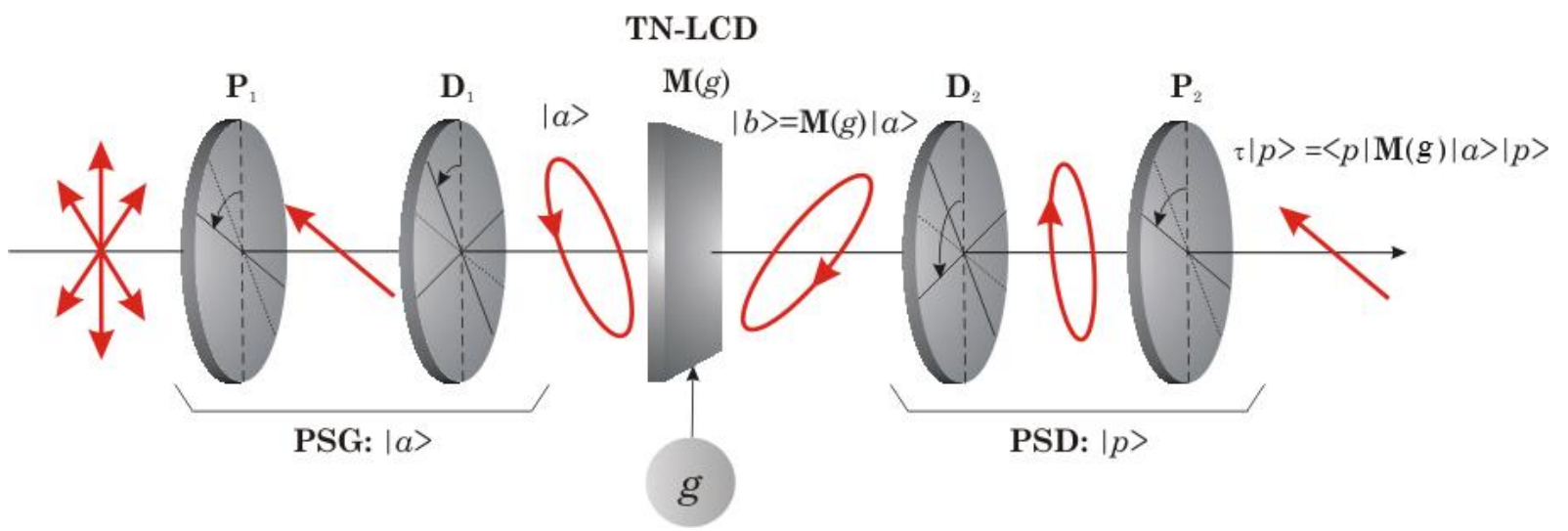

Fig. 4. Esquema de un sistema de modulación compleja. El TN-LCD se ilumina y detecta mediante estados elípticos para producir respuestas puras de fase con el nivel de gris.

\section{Modulación extendida de fase en pantallas TN-LCD}

En aplicaciones difractivas los moduladores TNLCD operan comúnmente mediante un esquema de iluminación y detección de luz mediante estados elípticos. Este tipo de esquema se muestra en la figura 4, donde se usa un generador de estados de polarización (PSG, del inglés Polarization State Generator) para iluminar el modulador y un detector de estados de polarización (PSD, del inglés Polarization State Detector) para detectar la luz emergente de éste. Al orientar de manera adecuada los elementos del PSD y el PSG (polarizadores lineales, P, y desfasadores de cuarto de onda, D) es posible producir modulaciones puras de fase en las que la intensidad media transmitida es prácticamente plana y sólo hay variación de la fase. Las configuraciones puras de fase son las deseadas comúnmente para reproducir hologramas o elementos difractivos de fase por su alta eficiencia. Otro aspecto, es que para codificar adecuadamente estos elementos, la intensidad transmitida no necesariamente debe ser el $100 \%$, sino que el requisito fundamental para controlar la luz difractada es que la amplitud sea lo más plana posible con el nivel de gris y la fase presente una variación de $2 \pi$ radianes. En las pantallas modernas, más delgadas, con poca capacidad de modulación de fase, las configuraciones habituales, como la de vectores propios rotados o autovectores [19], que producen una intensidad plana cerca del $100 \%$ no proporcionan una modulación de fase adecuada. Se observó en la literatura la existencia de otras configuraciones [20,21] que con menor intensidad producían mejor modulación de fase que la configuración de vectores propios. 


\section{$\begin{array}{ll}\text { (a) AUTOVECTORES } & \text { (b) } \mathbf{5 0} \%\end{array}$}

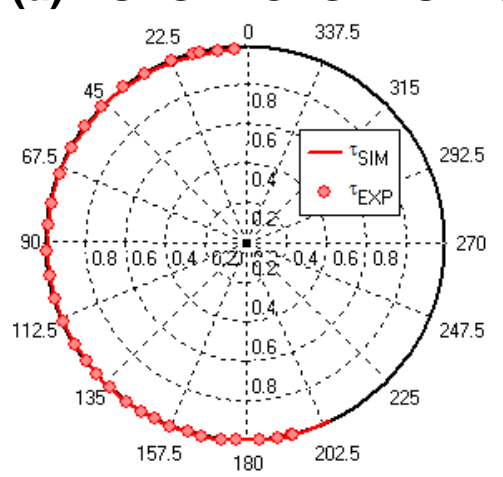

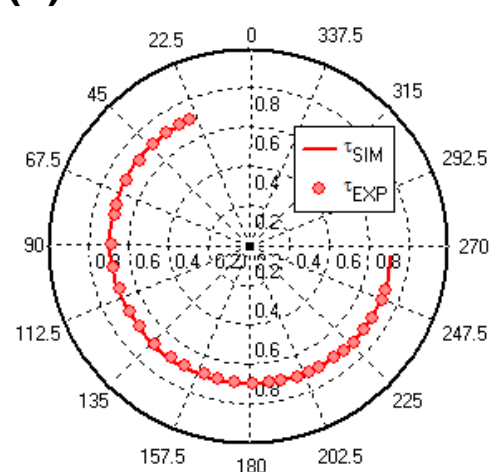

(c) $5 \%$

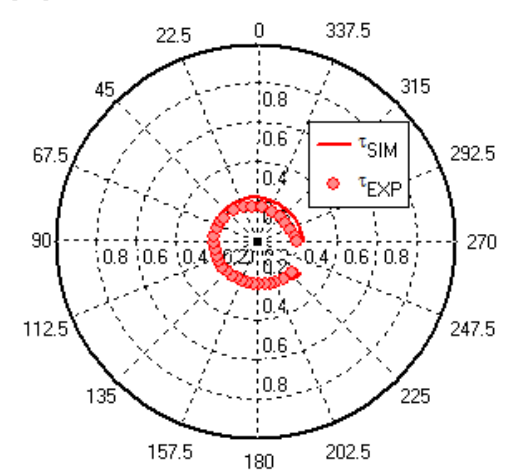

(d)

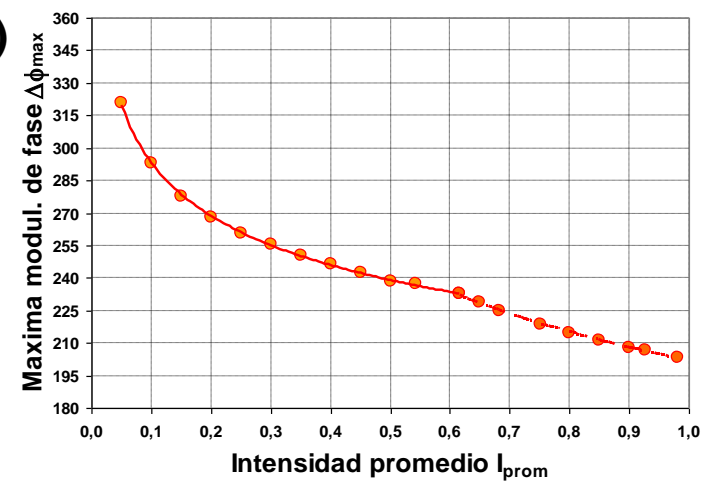

Fig. 5. Transmisión compleja para tres configuraciones de intensidad: (a) 100\% (autovectores), (b) 50\% de intensidad y (c) 5\% de intensidad. (d) Máxima modulación de fase en función de la intensidad media transmitida [4].

El hecho descrito, nos llevó a estudiar en detalle este fenómeno [4]. En particular, cómo evoluciona el rango de fase que se puede alcanzar en función del nivel de intensidad promedio. Para ello, se optimizaron los estados elípticos que iluminan y detectan el dispositivo y se comprobó cómo la modulación total de fase del sistema se podía aumentar progresivamente a costa de reducir la intensidad media transmitida [4]. Este fenómeno se pudo constatar experimentalmente en diversos dispositivos de este tipo. La Fig. 5 muestra cómo, en nuestro dispositivo, partiendo de la configuración de autovectores (Fig. 5(a)), con transmisión en intensidad cercana al $100 \%$, pero con modulación de fase de poco más de $\pi$ radianes, es posible alcanzar alrededor de $230^{\circ}$ para el $50 \%$ de intensidad transmitida, (Fig. 5 (b)) y prácticamente valor ideal de $2 \pi$ radianes a costa de reducir la intensidad transmitida hasta el 5\%, (Fig. 5(c)). Los resultados se obtuvieron empleando una longitud de onda de $514 \mathrm{~nm}$. Finalmente, la Fig. 5(d) muestra el comportamiento de la máxima modulación de fase obtenida en función de la intensidad media transmitida para diversas configuraciones optimizadas. Podemos observar cómo la fase presenta un tramo casi lineal hasta el $50 \%$ y luego crece de manera no lineal a medida que la intensidad se aproxima 0 .

\section{Estudio de la modulación compleja mediante la fase de Pancharatnam}

El fenómeno anterior motivó la aplicación conceptos como la fase geométrica o de Pancharatnam [6] con el objeto de explicar el comportamiento los sistemas de modulación compleja. La fase de Pancharatnam es una idea que, bajo las interpretaciones modernas, expresa la fase que gana un estado de polarización al recorrer un circuito cerrado en la esfera de Poincaré provocado por una serie de proyecciones sucesivas (pasos por polarizadores elípticos). Esta clase de sistemas en los que un estado retorna a su polarización inicial se denominan sistemas cíclicos. Así, la fase 
(a)

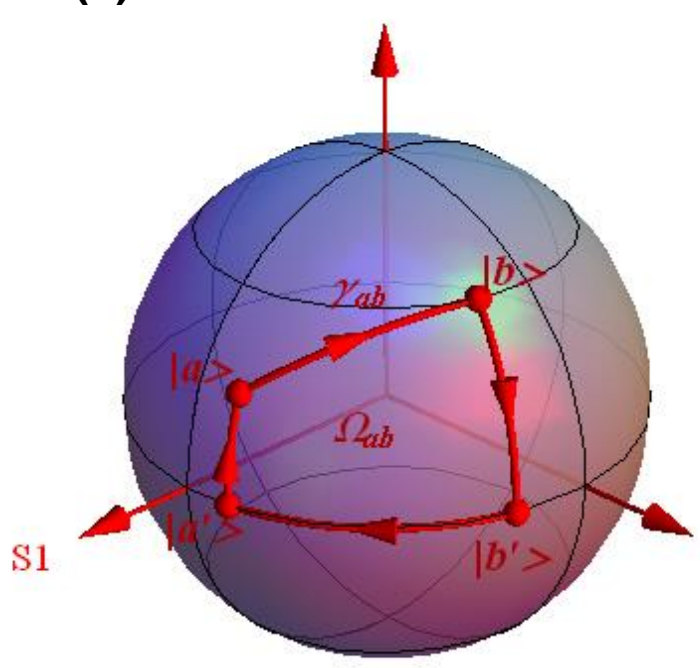

(b)

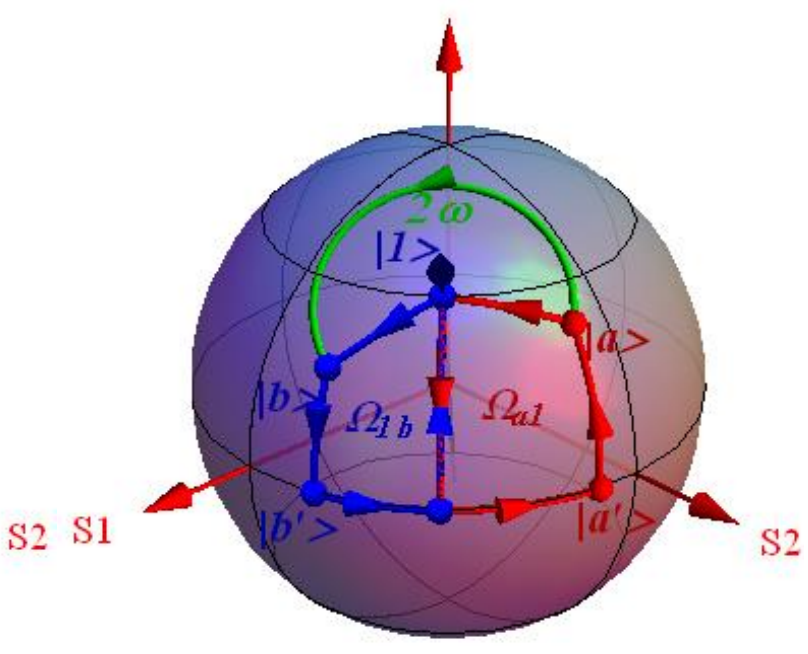

Fig. 6. (a) Acción de un polarizador elíptico y (b) un desfasador elíptico sobre la esfera de Poincaré.

geométrica ganada por un estado en un sistema cíclico iguala al negativo de la mitad del ángulo sólido encerrado por la trayectoria. Asimismo, si las transformaciones del estado de polarización incluyen el paso por elementos no absorbentes, tales como desfasadores elípticos (o equivalentemente, cuando los estados se mueven por arcos no geodésicos en la esfera), entonces es preciso añadir otro término de fase adicional denominado fase dinámica [22,23] o bien replantear la formulación del problema [24].

\section{4.a. Acción de polarizadores y desfasadores sobre la esfera}

$\mathrm{Si}$ se elige la siguiente representación de vectores de Jones para un estado con acimut $\theta$ y elipticidad $\varepsilon$ :

$$
\begin{aligned}
|\theta, \varepsilon\rangle & =\left[\begin{array}{cc}
\cos \theta & -\operatorname{sen} \theta \\
\operatorname{sen} \theta & \cos \theta
\end{array}\right]\left[\begin{array}{c}
\cos \varepsilon \\
i \operatorname{sen} \varepsilon
\end{array}\right]= \\
& =\mathbf{R}(-\theta)\left[\begin{array}{c}
\cos \varepsilon \\
i \operatorname{sen} \varepsilon
\end{array}\right],
\end{aligned}
$$

es posible demostrar que la proyección entre un estado $|a\rangle$ y un estado $|b\rangle$, que representa un polarizador elíptico (o bien cualquier otro estado sobre el que se proyecta) viene dada por:

$$
\langle a \mid b\rangle=\cos \left(\frac{\gamma_{a b}}{2}\right) \exp \left(-i \frac{\Omega_{a b}}{2}\right)
$$

siendo $\gamma_{a b}$ el arco geodésico que une ambos estados y $\Omega_{a b}$ el ángulo sólido que se obtiene al unir estos estados mediante arcos geodésicos entre sí y con el ecuador, tal y como se muestra en la Fig. 6(a). El signo de este ángulo es positivo si la secuencia $a \rightarrow b \rightarrow b^{\prime} \rightarrow a^{\prime} \rightarrow a$ es antihoraria y negativo si es horaria.

Considérese un desfasador elíptico representado por:

$$
\begin{aligned}
\mathbf{D} & \left.=\mathrm{e}^{i \phi_{1}}|1\rangle 1\left|+\mathrm{e}^{i \phi_{2}}\right| 2\right\rangle 2 \mid= \\
& \left.=\mathrm{e}^{i \Phi}\left[\mathrm{e}^{-i \omega}|1\rangle 1\left|+\mathrm{e}^{i \omega}\right| 2\right\rangle 2 \mid\right]
\end{aligned}
$$

donde $\phi_{1}$ y $\phi_{2}$ son los retardos de sus ejes neutros $|1\rangle$ y $|2\rangle$, respectivamente, y, por tanto, $2 \omega=\phi_{2}-\phi_{1}$ es su retardo y $\phi=\left(\phi_{2}+\phi_{1}\right) / 2$ su fase promedio. Cuando este dispositivo se ilumina mediante un estado $|a\rangle$, produce un estado de salida:

$$
|B\rangle=\exp \left(i \phi_{D: a \rightarrow b}\right)|b\rangle
$$

donde $|b\rangle$ es un estado unitario que se obtiene al rotar $|a\rangle$ una cantidad antihoraria $2 \omega$ sobre el eje $|1\rangle$ del desfasador y está representado según la notación dada en la Ec. (5), y donde $\phi_{D: a \rightarrow b}$ es el factor externo de fase introducido por el desfasador, que se ha de calcular como:

$$
\phi_{D: a \rightarrow b}=\phi_{1}-\frac{\Omega_{a 1}+\Omega_{1 b}}{2}
$$


donde $\Omega_{a 1}$ y $\Omega_{1 b}$ son los ángulos sólidos que representan la fase de las proyecciones $\langle a \mid 1\rangle \mathrm{y}$ $\langle 1 \mid b\rangle$, respectivamente. Todas estas magnitudes y transformaciones quedan representadas en la Fig. 6(b).

\section{4.b. Acción de un sistema de modulación compleja sobre la esfera}

Combinando las ideas expuestas en el apartado anterior, es posible llegar a una representación sobre la esfera de Poincaré de la acción de un sistema de modulación compleja completo como el de la Fig. 7(a). Es preciso notar que cualquier dispositivo no absorbente (modulador en este caso) se puede asimilar a un desfasador elíptico, que constituiría la parte central del sistema de modulación. El estado $|a\rangle$ que ilumina el desfasador se genera mediante un generador de estados de polarización o PSG (del inglés Polarization State Generator) constituido por un polarizador lineal y un desfasador $\lambda / 4$, mientras que el estado $|p\rangle$ por el que se proyecta la salida del modulador se define mediante la misma clase de elementos dispuestos en orden inverso constituyendo un detector de estados de polarización o PSD (Polarization State Detector)

La Fig. 7(b) representa toda la información necesaria para el cálculo de la transmisión compleja del sistema, tanto el módulo como la fase, sobre la esfera de Poincaré. Se puede demostrar que la transmisión compleja viene dada por la expresión

$$
\tau=\cos \frac{\gamma_{b p}}{2} \exp \left(i\left[\Phi-\frac{\Omega_{a p}}{2}-\frac{\Omega_{a b p+} \Omega_{a a b b}}{2}\right]\right)
$$

(a)

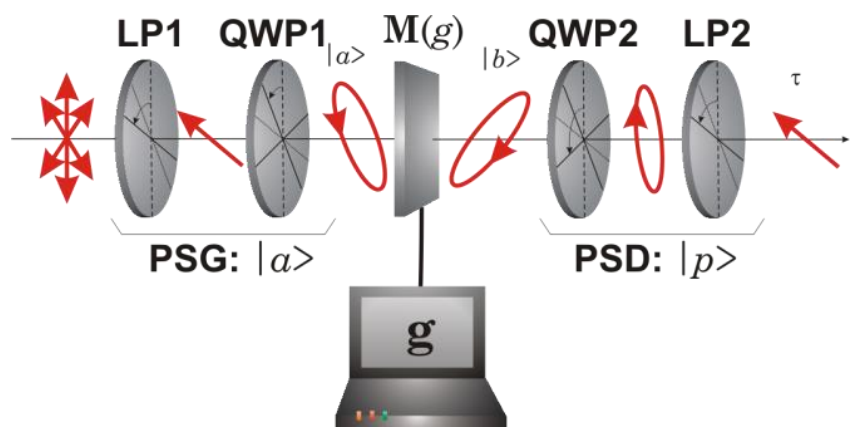

En esta expresión, $\gamma_{b p}$ es el arco geodésico entre el estado de salida del modulador y el estado que representa el detector de estados de polarización. Este factor controla la transmisión de amplitud del sistema. En cuanto a los términos que gobiernan la fase del sistema, $\phi$ es la fase promedio del modulador, que en general depende del nivel de gris (o del voltaje) aplicado, pero es completamente insensible a la configuración de polarización elegida. Por tanto, es un término de fase que no se representa sobre la esfera en este caso. $\Omega_{a p}$ es el ángulo sólido asociado a la proyección del estado de entrada sobre el estado detectado y es un término completamente constante con el nivel de gris (dado que estos estados son fijos en este problema), por lo que puede ser obviado. Por otro lado, $\Omega_{a b p}$ y $\Omega_{a a b b}$ son los ángulos sólidos encerrados en la esfera por las secuencias $a \rightarrow b \rightarrow p$ y $a \rightarrow a \rightarrow b \rightarrow b$, respectivamente, $\mathrm{y}$ son, en definitiva, los que determinan la contribución geométrica a la modulación de fase del sistema.

\section{4.c. Resultados experimentales}

A continuación se muestra un ejemplo experimental en el que se aplica el procedimiento descrito para predecir la modulación compleja provocada por un modulador PAL-LCD (del inglés Parallel Aligned LCD) cuando se ilumina mediante luz lineal a $45^{\circ}$ de su eje neutro $|1\rangle$ y se detecta también mediante luz lineal a $30^{\circ}$ de éste. El dispositivo es un modulador modelo X10468-01 de

Fig. 7. (a) Sistema de modulación compleja y (b) representación de las magnitudes involucradas en el cálculo de la transmitancia compleja sobre la esfera de Poincaré [8]. 

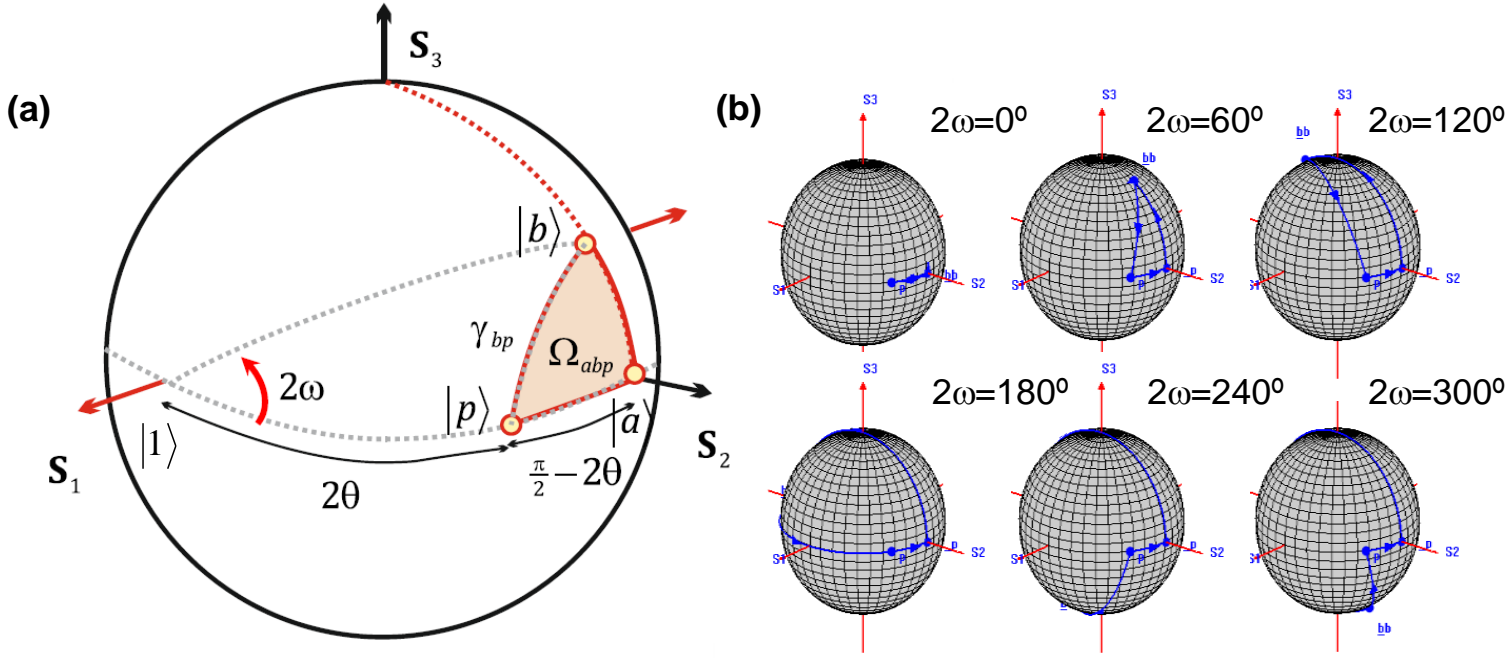

(c)

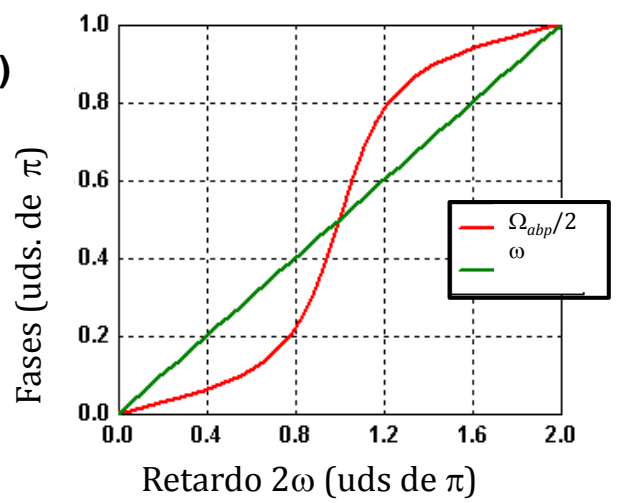

(d)

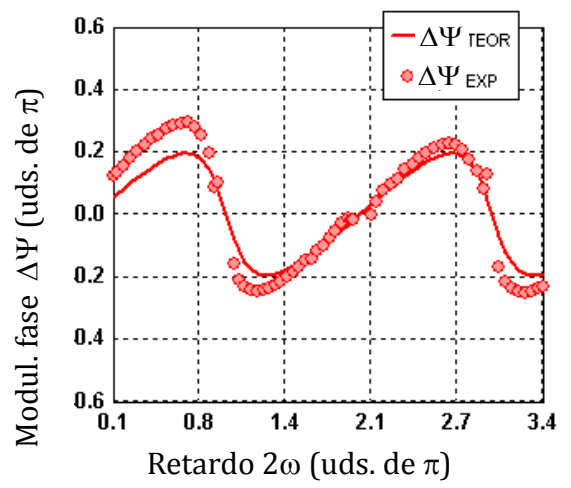

Fig. 8. (a) Magnitudes sobre la esfera involucradas en la fase geométrica del sistema experimental con el PAL. (b) representación sobre la esfera del triángulo esférico abp para distintos valores de retardo. (c) Mitad del ángulo sólido del triángulo abp y retardo del modulador. (d) Modulación de fase predicha (línea) y medida (puntos) [8].

Hamamatsu. La Fig. 8(a) muestra sobre la esfera de Poincaré las magnitudes involucradas en el cálculo de la fase. En este caso, se puede apreciar que $\Omega_{a a b b}=0$ dado que se ilumina el dispositivo a $45^{\circ}$ de sus ejes neutros. Por otro lado, la fase promedio en un PAL vale $\phi=\omega$. Por lo tanto, el único término geométrico que determinará la modulación de fase será el ángulo sólido $\Omega_{a b p}$, el cual se puede comprobar mediante trigonometría esférica que cumple:

$$
\tan \left(\Omega_{a b p}\right)=\frac{\operatorname{sen} 2 \omega \cos 2 \theta}{\cos 2 \omega+\operatorname{sen} 2 \theta}
$$

con $\theta=30^{\circ}$ la orientación del analizador. La Fig. 8(b) muestra la evolución del ángulo sólido sobre la esfera para distintos valores del retardo del dispositivo. En consecuencia, la modulación de fase $\Psi$ valdrá:

$$
\Psi=\omega-\frac{\Omega_{a b p}}{2}
$$

que es la contribución de la mitad del retardo menos la mitad del área del triángulo esférico abp. Ambos factores se representan en función del retardo en la Fig. 8(c).

Usando la configuración descrita, se envió una red de difracción binaria para evaluar la modulación de fase provocada por el dispositivo. La predicción teórica en base a argumentos geométricos (línea) y los valores medidos experimentalmente mediante la red (puntos) se representan en la Fig. 8(d). Se puede apreciar que existe una concordancia satisfactoria entre ambos que verifica la validez del procedimiento propuesto. 
(a)

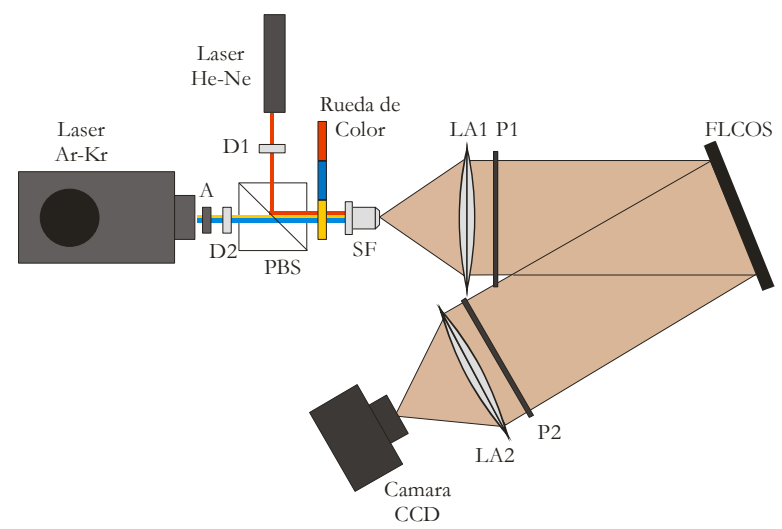

(c)

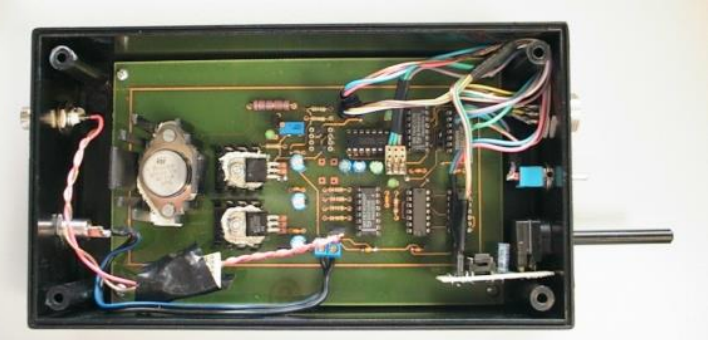

(b)

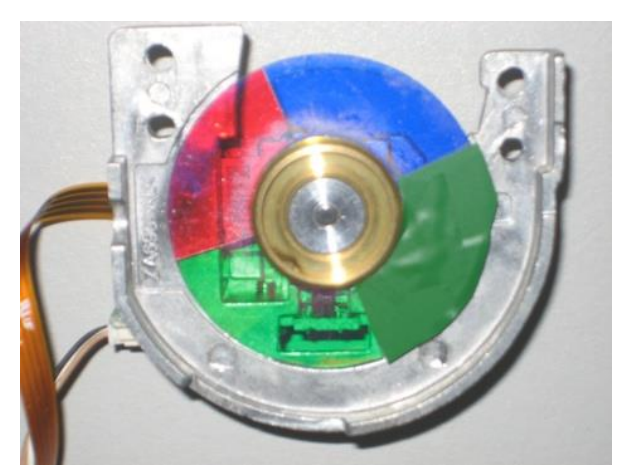

(d)

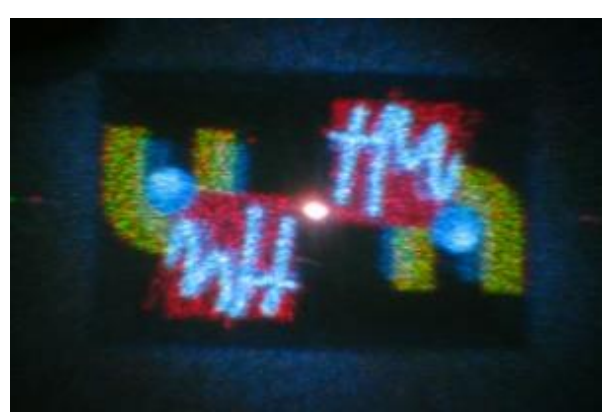

Fig. 9. (a) Representación esquemática del sistema de generación de hologramas en color. (b) rueda de filtros de color extraída de un video-proyector para realizar el filtrado de las líneas del láser. (c) Sistema electrónico de control de la rueda desarrollado. (d) Ejemplo de un holograma en color obtenido mediante el sistema descrito [9].

\section{Sistemas experimentales con moduladores espaciales de luz de cristal líquido}

En esta sección se presenta un breve resumen de los sistemas experimentales desarrollados en la tesis.

\section{5.a. Sistema de transformada de Fourier en color}

Este sistema [9] constituye un sistema óptico difractivo para reproducción de hologramas en color basado en un modulador de cristal líquido ferroeléctrico o FLCD (del inglés Ferroelectric Liquid Crystal Display), una rueda de filtros de color, extraída de un sistema de videoproyección DLP, y una iluminación láser de tres líneas (roja, verde y azul). Con el objeto de conseguir tres canales de color, se usan un láser multilínea de Ar-Kr (que aporta las líneas verde y azul) y un láser de $\mathrm{He}-\mathrm{Ne}$ (que aporta la línea roja). El sistema óptico empleado se muestra en la Fig. 9(a). La rueda de filtros, que se muestra en la Fig. 9(b), se sincroniza con las imágenes de fase enviadas al modulador mediante un programa y un sistema electrónico de control, que se muestra en la Fig. 9(c). Finalmente, la Fig. 9(d) muestra un ejemplo de reconstrucción de un holograma en color mediante este sistema, en el que las máscaras de fase de cada canal de color han sido obtenidas mediante un algoritmo iterativo IFTA [25].

\section{5.b. Sistema de correlación óptica en color}

El sistema anterior se adaptó para realizar un sistema de correlación óptica para imágenes en color [11] mediante una arquitectura de correlador de transformada conjunta o JTC (del inglés Joint Transform Correlator) [10], con el fin de adaptado al reconocimiento óptico de imágenes en color.

\section{5.c. Sistema de generación de patrones tridimensionales de puntos}

En este sistema [15], se utilizó un TN-LCD operando como modulador binario de fase, para realizar un sistema simple capaz de generar patrones de focalización de puntos tridimensionales de igual intensidad. Esta clase de patrones pueden resultar de utilidad en sistemas de foto-polimerización de compuestos orgánicos o en sistemas de micro-grabado de 
estructuras ópticas con láseres pulsados, pues el uso de moduladores elimina la necesidad de emplear elementos mecánicos en estos procesos y además permite crear varias estructuras simultáneamente.

\section{5.d. Sistema de codificación de imágenes mediante estados de polarización}

En este trabajo [16] se realizó un sistema sencillo basado en un modulador TN-LCD para la codificación óptica de información mediante estados de polarización, y demostrar su utilidad para realizar un sistema esteganográfico para la ocultación de información.

\section{Conclusiones}

En definitiva, se han resumido las principales contribuciones teóricas y prácticas desarrolladas en la tesis mencionada. En concreto se han aplicado modelos de dispersión de los parámetros microscópicos de moduladores TNLCD para la predicción espectral de su respuesta.
Se ha estudiado en detalle el fenómeno de la modulación extendida de fase y se han desarrollado herramientas de análisis geométrico de la modulación compleja sobre la esfera de Poincaré para moduladores ópticos. En cuanto al aspecto experimental, se han desarrollado sistemas de generación de hologramas en color, correlación óptica en color, generación de patrones tridimensionales de puntos y para la codificación de imágenes mediante estados de polarización, haciendo uso de las tres principales tecnologías de moduladores de cristal líquido.

\section{Agradecimientos}

Agradezco la concesión de una beca de carácter predoctoral por parte de la Generalitat Valenciana gracias a la cual pude desarrollar el trabajo descrito. Además deseo manifiestamente agradecer el apoyo de mis padres y el de todas aquellas personas cercanas durante este periodo. Apoyo sin el cual este trabajo habría sido imposible sin duda alguna. 